TRANSACTIONS OF THE

AMERICAN MATHEMATICAL SOCIETY

Volume 364, Number 5, May 2012, Pages 2261-2280

S 0002-9947(2011)05293-1

Article electronically published on December 16, 2011

\title{
GOLDEN TILINGS
}

\author{
A. A. PINTO, J. P. ALmEIDA, AND A. PORTELA
}

\begin{abstract}
We introduce the notion of golden tilings, and we prove a oneto-one correspondence between (i) smooth conjugacy classes of Anosov diffeomorphisms, with an invariant measure absolutely continuous with respect to the Lebesgue measure, (ii) affine classes of golden tilings and (iii) solenoid functions. The solenoid functions give a parametrization of the infinite dimensional space consisting of the mathematical objects described in the above equivalences.
\end{abstract}

\section{INTRODUCTION}

Inspired in the works of Y. Jiang [12], A. Pinto and D. Sullivan [28] and A. Pinto and D. Rand [19], we introduce the notion of golden tilings. The golden tilings record the infinitesimal geometric structure determined by the dynamics along the unstable leaf that is invariant by the Anosov diffeomorphism. We define the properties of the golden tilings using the Fibonacci decomposition of the natural numbers instead of the dyadic decomposition, because the Fibonacci decomposition has the advantage of encoding, in a natural way, the combinatorics determined by the Markov partition along the unstable leaf. Our goal is to exhibit a natural correspondence between golden tilings, Anosov diffeomorphisms and solenoid functions.

1.1. Main theorem. Let $\gamma=2 /(1+\sqrt{5})$ be the inverse of the golden number. The (golden) Anosov automorphism $G_{A}: \mathbb{T} \rightarrow \mathbb{T}$ is given by $G_{A}(x, y)=(x+y, x)$, where $\mathbb{T}$ is equal to $\mathbb{R}^{2} /(v \mathbb{Z} \times w \mathbb{Z})$ with $v=(\gamma, 1)$ and $w=(-1, \gamma)$. A $C^{1+}$ (golden) Anosov diffeomorphism $G: \mathbb{T} \rightarrow \mathbb{T}$ is a $C^{1+\alpha}$ diffeomorphism, with $\alpha>0$, such that (i) $G$ is topologically conjugate to $G_{A}$ by a map $h_{G}$; (ii) the tangent bundle has a $C^{1+\alpha}$ uniformly hyperbolic splitting into a stable direction and an unstable direction (see [30]). We denote by $\mathcal{G}$ the set of all such $C^{1+}$ Anosov diffeomorphisms with an invariant measure absolutely continuous with respect to the Lebesgue measure.

The eigenvalues of the Anosov automorphism $G_{A}$ are $\mu^{-}=-\gamma$ and $\mu^{+}=1 / \gamma$. Let $\pi: \mathbb{R}^{2} \rightarrow \mathbb{T}$ be the natural projection of $\mathbb{R}^{2}$ in $\mathbb{T}$. Let $\mathbf{A}_{0}$ and $\mathbf{B}_{0}$ be the rectangles $[0,1] \times[0,1]$ and $[-\gamma, 0] \times[0, \gamma]$, respectively (see Figure 1). A Markov partition $\mathcal{M}_{A}$ of $G_{A}$ is given by $\mathbf{A}=\pi\left(\mathbf{A}_{0}\right)$ and $\mathbf{B}=\pi\left(\mathbf{B}_{0}\right)$. The unstable manifolds of $G_{A}$ correspond to the projection by $\pi$ of the vertical lines of the plane, and the stable manifolds of $G_{A}$ are the projection by $\pi$ of the horizontal lines of the plane. Let $W_{0}$ be the positive vertical axis of $\mathbb{R}^{2}$. Hence $W=\pi\left(W_{0}\right)$ is the unstable leaf of $G_{A}$ with only one endpoint $y_{0}=\pi(0,0)$ that is the fixed point of $G_{A}$. The unstable

Received by the editors February 21, 2008 and, in revised form, January 5, 2010.

2000 Mathematics Subject Classification. Primary 37C15; Secondary 37C40.

Key words and phrases. Anosov diffeomorphisms, circle diffeomorphisms, dynamics, renormalization, solenoid functions, tilings. 
leaf $W$ passes first through all the unstable boundaries of the Markov rectangles A and B. Let the unstable spanning leaf segment $K_{1}$ be the left unstable boundary of the Markov rectangle A (see the definition of a spanning leaf segment in Section 2.1). Let the unstable spanning leaf segment $K_{2}$ be the left unstable boundary of the Markov rectangle B. Let $K_{3}, K_{4}, \ldots \in W$ be the unstable leaf segments defined, inductively, as follows: (i) $K_{i}$ is an unstable spanning leaf of a Markov rectangle, for every $i \geq 3$; (ii) $K_{i} \cap K_{i+1}=\left\{y_{i}\right\}$ is a common boundary point of both $K_{i}$ and $K_{i+1}$, for every $i \geq 2$ (see Figure 1). We note that $W=\bigcup_{i \geq 1} K_{i}$.

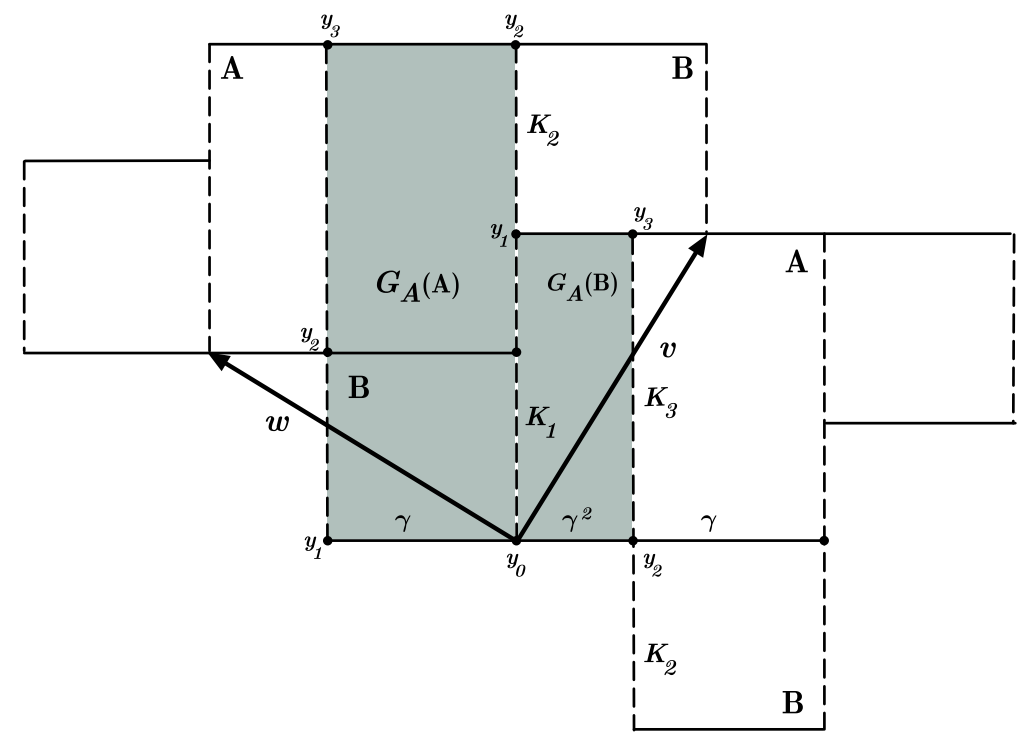

Figure 1. The golden automorphism $G_{A}$.

Theorem 1.1 (Flexibility). There is a well-defined map $G \rightarrow\left(a_{i}^{G}\right)_{i \in \mathbb{N}}$ that associates to each $C^{1+}$ Anosov diffeomorphism $G$ in $\mathcal{G}$ the golden sequence $\left(a_{i}^{G}\right)_{i \in \mathbb{N}}$ given by

$$
a_{i}^{G}=\lim _{n \rightarrow \infty} \frac{\left|G^{-n}\left(h_{G}\left(K_{i+1}\right)\right)\right|}{\left|G^{-n}\left(h_{G}\left(K_{i}\right)\right)\right|},
$$

where $|I|$ means the length of the unstable leaf segment I with respect to a Riemannian metric on $\mathbb{T}$. Furthermore, this map determines a one-to-one correspondence between smooth conjugacy classes of Anosov diffeomorphisms in $\mathcal{G}$ and golden sequences.

We leave the definition of the golden sequences to Section 3.7 and we will prove Theorem 1.1 in Section 3.8 ,

The Fibonacci numbers $F_{1}, F_{2}, F_{3}, \ldots$ are inductively given by the well-known relation $F_{n+2}=F_{n+1}+F_{n}$, with $n \geq 1$, where we choose $F_{1}=1$ and $F_{2}=2$. For any natural number $i \in \mathbb{N}$, we define the finite sequence $F_{n_{0}}, F_{n_{1}}, \ldots, F_{n_{p}}$ as follows: (i) $F_{n_{0}}$ is the largest Fibonacci less or equal to $i$; (ii) inductively, if $F_{n_{0}}+\cdots+F_{n_{k-1}}<$ $i$, then $F_{n_{k}}$ is the largest Fibonacci less than or equal to $i-\left(F_{n_{0}}+\cdots+F_{n_{k-1}}\right)$. We observe that there is an integer $p \in \mathbb{N}$ and a Fibonacci number $F_{n_{p}}$ such that

$$
i=F_{n_{0}}+\cdots+F_{n_{p}} \text {. }
$$


We call $F_{n_{0}}, \ldots, F_{n_{p}}$ the Fibonacci decomposition of $i \in \mathbb{N}$. We observe that every natural number $i \in \mathbb{N}$ has a unique Fibonacci decomposition.

Definition 1.2. The rigid golden sequence $\left(a_{i}^{R}\right)_{i \in \mathbb{N}}$ is defined as follows. For every $i \in \mathbb{N}$, with Fibonacci decomposition $F_{n_{0}}, \ldots, F_{n_{p}}$, we define

(i) $a_{i}^{R}=\gamma^{-1}$ if either $\left(n_{p}=1\right.$ and $n_{p-1}$ is odd) or ( $n_{p}=2$ and $n_{p-1}$ is even);

(ii) $a_{i}^{R}=\gamma$ if either $\left(n_{p}=1\right.$ and $n_{p-1}$ is even) or ( $n_{p}>2$ and $n_{p}$ is odd);

(iii) $a_{i}^{R}=1$ if either ( $n_{p}=2$ and $n_{p-1}$ is odd) or ( $n_{p}>2$ and $n_{p}$ is even).

In Theorem 1.1, we prove the existence of an infinite dimensional space of wellcharacterized golden sequences. However, the only golden sequence that we are able to make explicit is the rigid golden sequence.

Theorem 1.3 (Rigidity). Every Anosov diffeomorphism $G \in \mathcal{G}$, with a $C^{1+\text { zygmund }}$ complete system of unstable holonomies, determines a golden sequence $\left(a_{i}^{G}\right)_{i \in \mathbb{N}}$ that is rigid.

The definition of a $C^{1+z y g m u n d}$ complete system of unstable holonomies and the proof of Theorem 1.3 are given in Section 4

\section{Solenoid FunCTIONS}

Let $G$ be a $C^{1+}$ Anosov diffeomorphism in $\mathcal{G}$. We define the map $G_{\iota}=G$ if $\iota=u$ or $G_{\iota}=G^{-1}$ if $\iota=s$. For $\iota \in\{s, u\}$ and $x \in \mathbb{T}$, we denote the local $\iota$-manifolds through $x$ by

$$
W^{\iota}(x, \varepsilon)=\left\{y \in \mathbb{T}: d\left(G_{\iota}^{-n}(x), G_{\iota}^{-n}(y)\right) \leq \varepsilon, \text { for all } n \geq 0\right\},
$$

where $d$ is the distance determined by a Riemannian metric on the torus. By the Stable Manifold Theorem [30, these sets are respectively contained in the stable and unstable immersed manifolds

$$
W^{\iota}(x)=\bigcup_{n \geq 0} G_{\iota}^{n}\left(W^{\iota}\left(G_{\iota}^{-n}(x), \varepsilon_{0}\right)\right)
$$

which are the image of $C^{1+\alpha}$ immersions $\kappa_{\iota, x}: \mathbb{R} \rightarrow \mathbb{T}$, for some $0<\alpha \leq 1$ and some small $\varepsilon_{0}>0$. An open (resp. closed) ı-leaf segment $I$ is defined as a subset of $W^{\iota}(x)$ of the form $\kappa_{\iota, x}\left(I_{1}\right)$, where $I_{1}$ is an open (resp. closed) subinterval (non-empty) in $\mathbb{R}$. An $\iota$-leaf segment is either an open or closed $\iota$-leaf segment. The endpoints of an $\iota$-leaf segment $I=\kappa_{\iota, x}\left(I_{1}\right)$ are the points $\kappa_{\iota, x}(u)$ and $\kappa_{\iota, x}(v)$, where $u$ and $v$ are the endpoints of $I_{1}$. The interior of an $\iota$-leaf segment $I$ is the complement of its boundary. A map $c: I \rightarrow \mathbb{R}$ is an $\iota$-leaf chart of an $\iota$-leaf segment $I$ if $c$ is a homeomorphism onto its image.

2.1. Spanning leaf segments. One can find a small enough $\epsilon_{0}>0$ such that for every $0<\epsilon<\epsilon_{0}$ there is $\delta=\delta(\epsilon)>0$ with the property that, for all points $w, z \in \mathbb{T}$ with $d(w, z)<\delta, W^{u}(w, \epsilon)$ and $W^{s}(z, \epsilon)$ intersect in a unique point that we denote by

$$
[z, w]=W^{u}(w, \epsilon) \cap W^{s}(z, \epsilon) .
$$

A rectangle $R$ is a subset of $\mathbb{T}$ which is (i) closed under the bracket, i.e. $x, y \in R \Rightarrow$ $[x, y] \in R$, and (ii) proper, i.e. it is the closure of its interior in $\mathbb{T}$. If $\ell^{u}$ and $\ell^{s}$ are, respectively, unstable and stable closed leaf segments intersecting in a single point, then we denote by $\left[\ell^{u}, \ell^{s}\right]$ the set consisting of all points of the form $[z, w]$ with $z \in \ell^{u}$ and $w \in \ell^{s}$. We note that $\left[\ell^{u}, \ell^{s}\right]$ is a rectangle. Conversely, given 
a rectangle $R$, for each $x \in R$ there are closed unstable and stable leaf segments of $\mathbb{T}, \ell^{u}(x, R) \subset W^{u}(x)$ and $\ell^{s}(x, R) \subset W^{s}(x)$ such that $R=\left[\ell^{u}(x, R), \ell^{s}(x, R)\right]$. The leaf segments $\ell^{u}(x, R)$ and $\ell^{s}(x, R)$ are called, respectively, unstable and stable spanning leaf segments (see Figure 2).

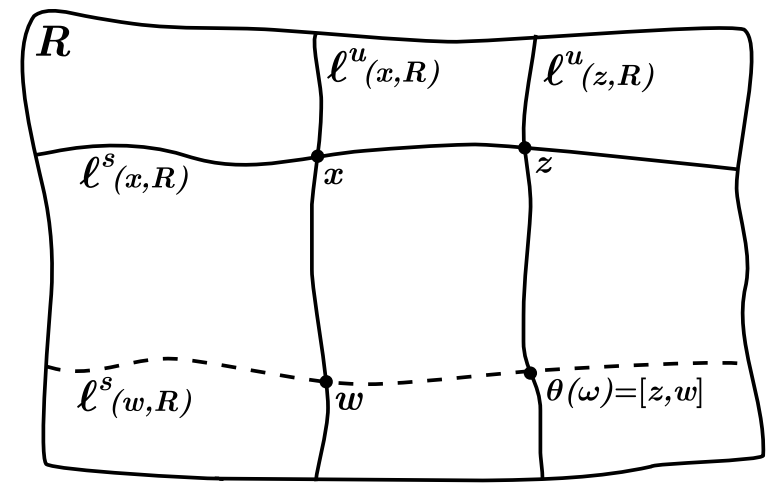

Figure 2. A basic unstable holonomy $\theta: \ell^{u}(x, R) \rightarrow \ell^{u}(z, R)$.

2.2. Basic holonomies. Suppose that $x$ and $z$ are two points inside any rectangle $R$ of $\mathbb{T}$. Let $\ell^{u}(x, R)$ and $\ell^{u}(z, R)$ be two unstable spanning leaf segments of $R$ containing, respectively, $x$ and $z$. We define the map $\theta: \ell^{u}(x, R) \rightarrow \ell^{u}(z, R)$ by $\theta(w)=[z, w]$ (see Figure 2). Such maps are called the basic unstable holonomies. They generate the pseudo-group of all unstable holonomies. Similarly, we can define the basic stable holonomies.

2.3. Lamination atlas. The unstable lamination atlas $\mathcal{L}=\mathcal{L}^{u}(G, \rho)$, determined by a Riemannian metric $\rho$, is the set of all maps $e: I \rightarrow \mathbb{R}$, where $e$ is an isometry between the induced Riemannian metric on the unstable leaf segment $I$ and the Euclidean metric on the reals. We call the maps $e \in \mathcal{L}$ the unstable lamination charts. By Theorem 2.1 in 23], the basic unstable and stable holonomies are $C^{1+}$ with respect to the lamination atlas $\mathcal{L}$ (see also [1] and [17).

2.4. HR structures. The HR structure associates an affine structure to each stable and unstable leaf segment of $G_{A}$ in such a way that these vary Hölder continuously with the leaf and are invariant under $G_{A}$ (see [24]).

An affine structure on a stable or unstable leaf of $G_{A}$ is equivalent to a ratio function $r(I: J)$ which can be thought of as prescribing the ratio of the size of two leaf segments $I$ and $J$ in the same stable or unstable leaf. A ratio function $r(I: J)$ is positive (we recall that each leaf segment has at least two distinct points) and continuous in the endpoints of $I$ and $J$. Moreover,

$$
r(I: J)=r(J: I)^{-1} \text { and } r\left(I_{1} \cup I_{2}: K\right)=r\left(I_{1}: K\right)+r\left(I_{2}: K\right),
$$

provided $I_{1}$ and $I_{2}$ intersect in at most one of their endpoints.

We say that $r$ is an unstable ratio function if (i) for all unstable leaf segments $K$ and for all unstable leaf segments $I, J \subset K, r(I: J)$ defines a ratio function on $K$; (ii) $r$ is invariant under $G_{A}$, i.e. $r(I: J)=r\left(G_{A}(I): G_{A}(J)\right)$ for all unstable leaf segments; and (iii) for every basic unstable holonomy map $\theta: I \rightarrow J$ between the unstable leaf segment $I$ and the unstable leaf segment $J$, defined with respect to 
a rectangle $R$, and for every unstable leaf segment $I_{0} \subset I$ and every unstable leaf segment $I_{1} \subset I$,

$$
\left|\log \frac{r\left(\theta I_{0}: \theta I_{1}\right)}{r\left(I_{0}: I_{1}\right)}\right| \leq \mathcal{O}\left((d(I, J))^{\epsilon}\right),
$$

where $\epsilon \in(0,1)$ depends upon $R$ and the constant of proportionality also depends upon $R$, but not on the segments considered. Since $r$ satisfies condition (2.2) and defines an affine structure on each leaf that is invariant under $G_{A}$, we say that $r$ is a tranversely Hölder unstable ratio function. An HR-structure is a pair $\left(r^{s}, r^{u}\right)$ consisting of a stable and an unstable ratio function.

2.5. Realized HR structures. Let $G$ be a $C^{1+}$ Anosov diffeomorphism in $\mathcal{G}$, and let $\mathcal{L}^{u}(G, \rho)$ be an unstable lamination atlas associated to a Riemannian metric $\rho$. If $I$ is an unstable leaf segment, then by $|I|$ we mean the length of the unstable leaf containing $I$ measured using the Riemannian metric $\rho$. Let $h_{G}: \mathbb{T} \rightarrow \mathbb{T}$ be the topological conjugacy between the automorphism $G_{A}$ and the Anosov diffeomorphism $G$. Using the mean value theorem and the fact that $G$ is $C^{1+\alpha}$ uniformly hyperbolic, for some $\alpha>0$, for all short unstable leaf segments $K$ of $G_{A}$ and all leaf segments $I$ and $J$ contained in $K$, the unstable realized ratio function $r_{G}^{u}$ given by

$$
r_{G}^{u}(I: J)=\lim _{n \rightarrow \infty} \frac{\left|G^{-n}\left(h_{G}(J)\right)\right|}{\left|G^{-n}\left(h_{G}(I)\right)\right|}
$$

is well defined (see Lemma 3.6 in 24]). Similarly, we have the definition of the stable realized ratio function $r_{G}^{s}$.

Lemma 2.1. The map $G \rightarrow r_{G}^{u}$ determines a one-to-one correspondence between $C^{1+}$ conjugacy classes of Anosov diffeomorphisms $G$ in $\mathcal{G}$ and unstable ratio functions.

Proof. By Theorem 5.1 in [24], there is a one-to-one correspondence $G \rightarrow\left(r_{G}^{s}, r_{G}^{u}\right)$ between $C^{1+}$ conjugacy classes of Anosov diffeomorphisms $G$ and HR-structures $\left(r_{G}^{s}, r_{G}^{u}\right)$. By Lemma 5 in [19], the unstable ratio function $r_{G}^{u}$ determines, uniquely, the stable ratio function $r_{G}^{s}$ if the Anosov diffeomorphism $G$ has an invariant measure that is absolutely continuous with respect to the Lebesgue measure (see also [3]).

2.6. Realizable solenoid functions. Let sol denote the set of all ordered pairs $(I, J)$ of unstable spanning leaf segments of the Markov rectangles $\mathbf{A}$ and $\mathbf{B}$ of $G_{A}$ such that the intersection of $I$ and $J$ consists of a single endpoint. Since the set sol is topologically a finite disjoint union of disjoint intervals, i.e. the disjoint union of a primary stable leaf of $\mathbf{A}$ and a primary stable leaf of $\mathbf{B}$, it has a natural topological structure.

We define a pseudo-metric $d_{\text {sol }}:$ sol $\times$ sol $\rightarrow \mathbb{R}^{+}$on the set sol by

$$
d_{\text {sol }}\left((I, J),\left(I^{\prime}, J^{\prime}\right)\right)=\max \left\{d\left(I, I^{\prime}\right), d\left(J, J^{\prime}\right)\right\} .
$$

Let $G$ be a $C^{1+}$ Anosov diffeomorphism in $\mathcal{G}$. We refer to the restriction $r_{G}^{u} \mid$ sol of an unstable ratio function $r_{G}^{u}$ to sol as the unstable realized solenoid function, and we denote it by $\sigma_{G}=r_{G}^{u} \mid$ sol. By construction, the restriction $\sigma_{G}$ of the unstable ratio function to sol gives a Hölder continuous function satisfying the matching condition and the boundary condition, which we now proceed to describe (see Theorem 6.1 in [24]). 
2.7. Hölder continuity of solenoid functions. The Hölder continuity of solenoid functions means that for all $t=(I, J)$ and $t^{\prime}=\left(I^{\prime}, J^{\prime}\right)$ in sol,

$$
\left|\sigma_{G}(t)-\sigma_{G}\left(t^{\prime}\right)\right| \leq \mathcal{O}\left(\left(d_{\text {sol }}\left(t, t^{\prime}\right)\right)^{\alpha}\right),
$$

for some $\alpha>0$.

2.8. Matching condition. Let $(I, J) \in$ sol. Suppose that there are pairs

$$
\left(I_{0}, I_{1}\right),\left(I_{1}, I_{2}\right), \ldots,\left(I_{n-2}, I_{n-1}\right) \in \text { sol }
$$

such that $G_{A} I=\bigcup_{j=0}^{k-1} I_{j}$ and $G_{A} J=\bigcup_{j=k}^{n-1} I_{j}$. Then

$$
\frac{\left|G_{A} J\right|}{\left|G_{A} I\right|}=\frac{\sum_{j=k}^{n-1}\left|I_{j}\right|}{\sum_{j=0}^{k-1}\left|I_{j}\right|}=\frac{\sum_{j=k}^{n-1} \prod_{i=1}^{j}\left|I_{i}\right| /\left|I_{i-1}\right|}{1+\sum_{j=1}^{k-1} \prod_{i=1}^{j}\left|I_{i}\right| /\left|I_{i-1}\right|} .
$$

Hence, the realized solenoid function $\sigma_{G}$ must satisfy the matching condition (see Figure 3) for all such leaf segments:

$$
\sigma_{G}(I: J)=\frac{\sum_{j=k}^{n-1} \prod_{i=1}^{j} \sigma_{G}\left(I_{i-1}: I_{i}\right)}{1+\sum_{j=1}^{k-1} \prod_{i=1}^{j} \sigma_{G}\left(I_{i-1}: I_{i}\right)} .
$$

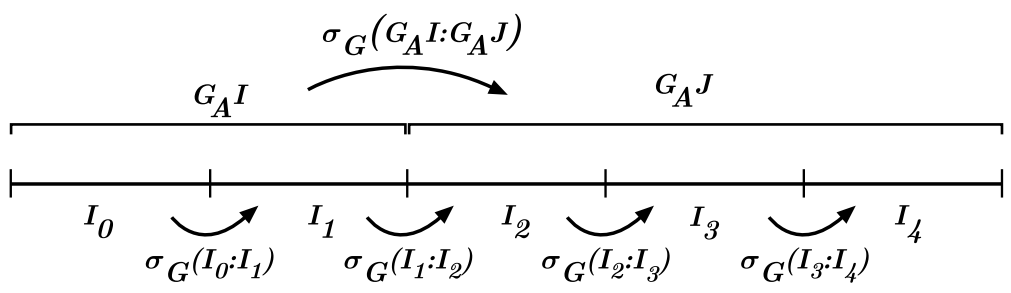

Figure 3. The matching condition for the solenoid function $\sigma_{G}$ with $k=2$ and $n=5$.

Lemma 2.2. Let $\sigma_{G}:$ sol $\rightarrow \mathbb{R}^{+}$be a realized solenoid function. The matching condition holds for $\sigma_{G}$ if, for every $\left(K_{1}, K_{2}\right) \in \mathbf{s o l}$, the following conditions hold:

(i) If $K_{1}, K_{2} \in \mathbf{A}$, then

$$
\sigma_{G}\left(K_{1}: K_{2}\right)=\frac{\sigma_{G}\left(I_{1}: I_{2}\right) \sigma_{G}\left(I_{2}: I_{3}\right)\left(1+\sigma_{G}\left(I_{3}: I_{4}\right)\right)}{1+\sigma_{G}\left(I_{1}: I_{2}\right)},
$$

where $I_{1}, I_{2}, I_{3}$ and $I_{4}$ are such that $G_{A}\left(K_{1}\right)=I_{1} \cup I_{2}, G_{A}\left(K_{2}\right)=I_{3} \cup I_{4}$ and $\left(I_{i}, I_{i+1}\right) \in$ sol for $i \in\{1,2,3\}$.

(ii) If $K_{1} \in \mathbf{A}$ and $K_{2} \in \mathbf{B}$, then

$$
\sigma_{G}\left(K_{1}: K_{2}\right)=\frac{\sigma_{G}\left(I_{1}: I_{2}\right) \sigma_{G}\left(I_{2}: I_{3}\right)}{1+\sigma_{G}\left(I_{1}: I_{2}\right)},
$$

where $I_{1}, I_{2}$ and $I_{3}$ are such that $G_{A}\left(K_{1}\right)=I_{1} \cup I_{2}, G_{A}\left(K_{2}\right)=I_{3}$ and $\left(I_{i}, I_{i+1}\right) \in$ sol for $i \in\{1,2\}$.

(iii) If $K_{1} \in \mathbf{B}$ and $K_{2} \in \mathbf{A}$, then

$$
\sigma_{G}\left(K_{1}: K_{2}\right)=\sigma_{G}\left(I_{1}: I_{2}\right)\left(1+\sigma_{G}\left(I_{2}: I_{3}\right)\right),
$$

where $I_{1}, I_{2}$ and $I_{3}$ are such that $G_{A}\left(K_{1}\right)=I_{1}, G_{A}\left(K_{2}\right)=I_{2} \cup I_{3}$ and $\left(I_{i}, I_{i+1}\right) \in$ sol for $i \in\{1,2\}$. 
Proof. If $\left(K_{1}, K_{2}\right) \in$ sol, then $\left(K_{1}, K_{2}\right)$ satisfies either condition $(i),(i i)$ or $(i i i)$ above (see Figure 7). Let us check that the formulas (2.5), (2.6) and (2.7) correspond to the matching condition (2.4) for $\sigma_{G}$ :

(i) If $K_{1}, K_{2} \in \mathbf{A}$, then there exists $\left(I_{i}, I_{i+1}\right) \in \mathbf{s o l}$, for $i=1,2,3$, such that $G_{A}\left(K_{1}\right)=I_{1} \cup I_{2}$ and $G_{A}\left(K_{2}\right)=I_{3} \cup I_{4}$. Furthermore,

$$
\frac{\left|G_{A}\left(K_{2}\right)\right|}{\left|G_{A}\left(K_{1}\right)\right|}=\frac{\left|I_{3}\right|+\left|I_{4}\right|}{\left|I_{1}\right|+\left|I_{2}\right|}=\frac{\left|I_{2}\right|}{\left|I_{1}\right|} \mid \frac{I_{3} \mid}{\left|I_{2}\right|}\left(1+\frac{\left|I_{4}\right|}{\left|I_{3}\right|}\right)\left(1+\frac{\left|I_{2}\right|}{\left|I_{1}\right|}\right)^{-1} .
$$

Hence, equality (2.5) follows from equality (2.4).

(ii) If $K_{1} \in \mathbf{A}$ and $K_{2} \in \mathbf{B}$, then there exists $\left(I_{i}, I_{i+1}\right) \in$ sol, for $i=1,2$, such that $G_{A}\left(K_{1}\right)=I_{1} \cup I_{2}$ and $G_{A}\left(K_{2}\right)=I_{3} \cup I_{4}$. Furthermore,

$$
\frac{\left|G_{A}\left(K_{2}\right)\right|}{\left|G_{A}\left(K_{1}\right)\right|}=\frac{\left|I_{3}\right|}{\left|I_{1}\right|+\left|I_{2}\right|}=\frac{\left|I_{2}\right|}{\left|I_{1}\right|} \frac{\left|I_{3}\right|}{\left|I_{2}\right|}\left(1+\frac{\left|I_{2}\right|}{\left|I_{1}\right|}\right)^{-1} \text {. }
$$

Hence, equality (2.6) follows from equality (2.4).

(iii) If $K_{1} \in \mathbf{B}$ and $K_{2} \in \mathbf{A}$, then there exists $\left(I_{i}, I_{i+1}\right) \in \mathbf{s o l}$, for $i=1,2$, such that $G_{A}\left(K_{1}\right)=I_{1}$ and $G_{A}\left(K_{2}\right)=I_{2} \cup I_{3}$. Furthermore,

$$
\frac{\left|G_{A}\left(K_{2}\right)\right|}{\left|G_{A}\left(K_{1}\right)\right|}=\frac{\left|I_{2}\right|+\left|I_{3}\right|}{\left|I_{1}\right|}=\frac{\left|I_{2}\right|}{\left|I_{1}\right|}\left(1+\frac{\left|I_{3}\right|}{\left|I_{2}\right|}\right) .
$$

Hence, equality (2.7) follows from equality (2.4).

2.9. Boundary condition. Let $\left(I_{i}, I_{i+1}\right),\left(J_{j}, J_{j+1}\right) \in$ sol, for each $i \in\{0, \ldots, m\}$ and each $j \in\{0, \ldots, n\}$ with the following properties: (i) $I_{0}=J_{0}$, (ii) $\bigcup_{i=1}^{m} I_{i}=$ $\bigcup_{j=1}^{m} J_{j}$ and (iii) $I_{i} \neq J_{j}$ for all $i \geq 1$ and all $j \geq 1$. Then the following two ratios are equal:

$$
\sum_{i=1}^{m} \prod_{j=1}^{i} \frac{\left|I_{j}\right|}{\left|I_{j-1}\right|}=\frac{\left|\bigcup_{i=1}^{m} I_{i}\right|}{\left|I_{0}\right|}=\frac{\left|\bigcup_{i=1}^{n} J_{j}\right|}{\left|J_{0}\right|}=\sum_{i=1}^{n} \prod_{j=1}^{i} \frac{\left|J_{j}\right|}{\left|J_{j-1}\right|} .
$$

We observe that the unstable spanning leaf segments $I_{1}, \ldots, I_{m}$ and $J_{1}, \ldots, J_{n}$ must be boundaries of Markov rectangles. Thus, a realized solenoid function $\sigma_{G}$ must satisfy the following boundary condition for all such leaf segments:

$$
\sum_{i=1}^{m} \prod_{j=1}^{i} \sigma_{G}\left(I_{j-1}: I_{j}\right)=\sum_{i=1}^{n} \prod_{j=1}^{i} \sigma_{G}\left(J_{j-1}: J_{j}\right) .
$$

Let $K_{1}, K_{2}$ and $K_{3}$ be the unstable spanning leaf segments as defined in Section 1.1. Let $K_{0}$ be the unstable spanning leaf segment in $A$ such that $K_{0} \cap K_{1}=\left\{y_{0}\right\}$. Let the unstable spanning leaf segment $I_{1}$ be the right boundary of the Markov rectangle $B$ and let the unstable spanning leaf segment $I_{2}$ be the right boundary of the Markov rectangle $A$ (see Figure 4).

Lemma 2.3. Let $\sigma_{G}:$ sol $\rightarrow \mathbb{R}^{+}$be a realized solenoid function. The boundary condition holds for $\sigma_{G}$ if the following conditions hold:

$$
\sigma_{G}\left(K_{0}: K_{1}\right)\left(1+\sigma_{G}\left(K_{1}: K_{2}\right)\right)=\sigma_{G}\left(K_{0}: I_{1}\right)\left(1+\sigma_{G}\left(I_{1}: I_{2}\right)\right)
$$

and

$$
\sigma_{G}\left(K_{3}: K_{2}\right)\left(1+\sigma_{G}\left(K_{2}: K_{1}\right)\right)=\sigma_{G}\left(K_{3}: I_{2}\right)\left(1+\sigma_{G}\left(I_{2}: I_{1}\right)\right) .
$$


Proof. Since $I_{1}$ and $K_{2}$ are the unstable boundaries of the Markov rectangle B and $I_{2}$ and $K_{1}$ are the unstable boundaries of the Markov rectangle $\mathbf{A}$, then the boundary condition (2.8) corresponds to

$$
\frac{\left|K_{1}\right|}{\left|K_{0}\right|}\left(1+\frac{\left|K_{2}\right|}{\left|K_{1}\right|}\right)=\frac{\left|K_{1} \cup K_{2}\right|}{\left|K_{0}\right|}=\frac{\left|I_{1} \cup I_{2}\right|}{\left|K_{0}\right|}=\frac{\left|I_{1}\right|}{\left|K_{0}\right|}\left(1+\frac{\left|I_{2}\right|}{\left|I_{1}\right|}\right)
$$

and

$$
\frac{\left|K_{2}\right|}{\left|K_{3}\right|}\left(1+\frac{\left|K_{1}\right|}{\left|K_{2}\right|}\right)=\frac{\left|K_{1} \cup K_{2}\right|}{\left|K_{3}\right|}=\frac{\left|I_{1} \cup I_{2}\right|}{\left|K_{3}\right|}=\frac{\left|I_{2}\right|}{\left|K_{3}\right|}\left(1+\frac{\left|I_{1}\right|}{\left|I_{2}\right|}\right) .
$$

Hence, the boundary condition for $\sigma_{G}$ is given by (2.9) and (2.10).

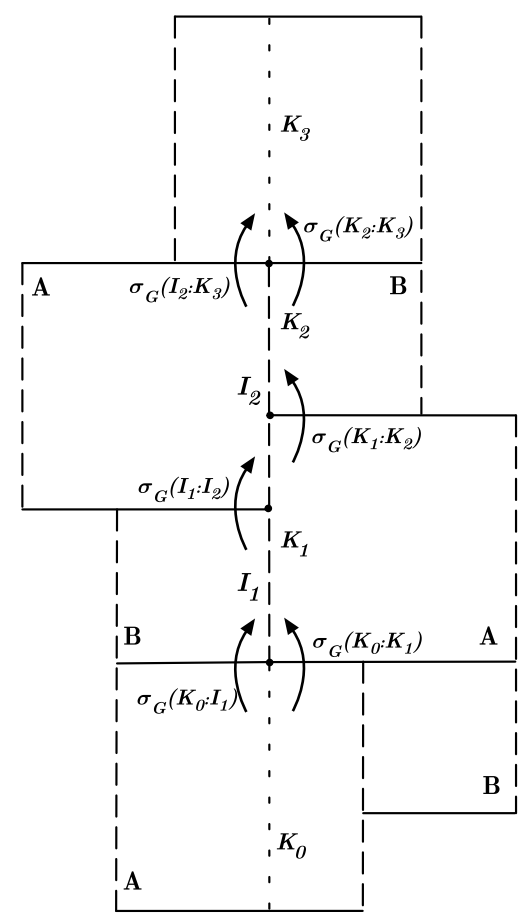

Figure 4 . The boundary condition for the realized solenoid function $\sigma_{G}$.

\subsubsection{Solenoid functions.}

Definition 2.4. A function $\sigma: \mathrm{sol} \rightarrow \mathbb{R}^{+}$is an (unstable) solenoid function if the following conditions hold: (i) $\sigma$ is Hölder continuous; (ii) $\sigma$ satisfies the matching condition given by the equalities (2.5), (2.6) and (2.7); and (iii) $\sigma$ satisfies the boundary condition given by the equalities (2.9) and (2.10).

By Theorem 6.1 in [24], we have the following equivalence:

Lemma 2.5. The map $r \rightarrow r \mid$ sol gives a one-to-one correspondence between ratio functions and solenoid functions.

Let $\mathcal{S O L}$ be the set consisting of all (unstable) solenoid functions. The set $\mathcal{S O L}$ has a natural metric. Combining Lemma 2.1 and Lemma 2.5, we obtain the following corollary. 
Corollary 2.6. The map $G \rightarrow r_{G} \mid$ sol determines a one-to-one correspondence between $C^{1+}$ conjugacy classes of Anosov diffeomorphisms $G$ in $\mathcal{G}$ and solenoid functions $r_{G} \mid$ sol in $\mathcal{S O} \mathcal{L}$.

\section{Golden tilings}

Recall from Section 1.1 the definitions of the unstable leaf segments $K_{1}, K_{2}, \ldots$, and the unstable leaf $W=\bigcup_{i \geq 1} K_{i}$. By construction, the set

$$
\mathcal{L}=\left\{\left(K_{i}, K_{i+1}\right), i \in \mathbb{N}\right\}
$$

is contained in sol and is dense in sol.

\subsection{Realized golden sequences.}

Lemma 3.1. There is a well-defined map $G \rightarrow\left(a_{i}^{G}\right)_{i \in \mathbb{N}}$ that associates to each $C^{1+}$ Anosov diffeomorphism $G$ in $\mathcal{G}$ the sequence $\left(a_{i}^{G}\right)_{i \in \mathbb{N}}$ given by

$$
a_{i}^{G}=\lim _{n \rightarrow \infty} \frac{\left|G^{-n}\left(h_{G}\left(K_{i+1}\right)\right)\right|}{\left|G^{-n}\left(h_{G}\left(K_{i}\right)\right)\right|} .
$$

Furthermore, $a_{i}^{G}=\sigma_{G}\left(K_{i}: K_{i+1}\right)$.

Recall that $h_{G}$ is the topological conjugacy between $G$ and the Anosov automorphism $G_{A}$.

Proof. By Lemma 2.5 and equation (2.3) we get that $\sigma_{G}\left(K_{i}: K_{i+1}\right)=r_{G}^{u}\left(K_{i}\right.$ : $\left.K_{i+1}\right)$, where

$$
r_{G}^{u}\left(K_{i}: K_{i+1}\right)=\lim _{n \rightarrow \infty} \frac{\left|G^{-n}\left(h_{G}\left(K_{i+1}\right)\right)\right|}{\left|G^{-n}\left(h_{G}\left(K_{i}\right)\right)\right|}
$$

is well defined. Since, by construction, $a_{i}^{G}=r_{G}^{u}\left(K_{i}: K_{i+1}\right)$, we get that $a_{i}^{G}$ is well defined and $a_{i}^{G}=\sigma_{G}\left(K_{i}: K_{i+1}\right)$.

\subsection{Fibonacci marking of the unstable leaf.}

Lemma 3.2. For every $i \in \mathbb{N}$ with Fibonacci decomposition $F_{n_{0}}, \ldots, F_{n_{p}}$ the following conditions hold:

(i) $K_{i} \in \mathbf{B}$ and $K_{i+1} \in \mathbf{A}$ if either $\left(n_{p}=1\right.$ and $n_{p-1}$ is odd) or $\left(n_{p}=2\right.$ and $n_{p-1}$ is even);

(ii) $K_{i} \in \mathbf{A}$ and $K_{i+1} \in \mathbf{B}$ if either ( $n_{p}=1$ and $n_{p-1}$ is even) or ( $n_{p}>2$ and $n_{p}$ is odd);

(iii) $K_{i}, K_{i+1} \in \mathbf{A}$ if either $\left(n_{p}=2\right.$ and $n_{p-1}$ is odd) or $\left(n_{p}>2\right.$ and $n_{p}$ is even).

Proof. Let $\pi: \mathbb{R}^{2} \rightarrow \mathbb{T}$ be the natural projection, where $\mathbb{T}=\mathbb{R}^{2} /(v \mathbb{Z} \times w \mathbb{Z})$. Let $\mathbb{S}=\mathbb{R} /[1+\gamma] \mathbb{Z}$ be the clockwise oriented circle with the metric induced by the Euclidean metric on $\mathbb{R}$. Let $\pi_{\mathbb{S}}: \mathbb{R} \rightarrow \mathbb{S}$ be the natural projection. The projection $\pi_{\mathbb{S}}$ has the property that

$$
\pi_{\mathbb{S}}(x)=\pi_{\mathbb{S}}(x+1+\gamma),
$$

for every $x \in \mathbb{R}$. Let $i_{\mathbb{S}}: \mathbb{S} \rightarrow \mathbb{T}$ be the natural inclusion. The inclusion $i_{\mathbb{S}}$ has the property that

$$
\pi(x, 0)=i_{\mathbb{S}} \circ \pi_{\mathbb{S}}(x),
$$

for every $x \in \mathbb{R}$. Recall that $K_{0}$ is the unstable spanning leaf segment such that $K_{0} \cap K_{1}=\left\{y_{0}\right\}$, where $y_{0}=\pi(0,0)$, and let $K_{1}, K_{2}, \ldots$ be the unstable spanning 
leaf segments such that $W=\bigcup_{i>1} K_{i}$ (see Section 1.1). For every $i \in \mathbb{N}_{0}$, (i) let $y_{i} \in \mathbb{T}$ be the point given by $\left\{y_{i}\right\}=K_{i} \cap K_{i+1}$; (ii) let $z_{i}=i_{\mathbb{S}}^{-1}\left(y_{i}\right)$; and (iii) let $w_{i} \in[-1, \gamma]$ be such that $\pi_{\mathbb{S}}\left(w_{i}\right)=z_{i}$. Hence, for every $i \in \mathbb{N}_{0}$ (see Figure [5),

(i) if $w_{i} \in(-\gamma, 0)$, then $K_{i} \in \mathbf{A}$ and $K_{i+1} \in \mathbf{B}$;

(ii) if $w_{i} \in\left(0, \gamma^{2}\right)$, then $K_{i}, K_{i+1} \in \mathbf{A}$;

(iii) if $w_{i} \in[-1,-\gamma) \cup\left(\gamma^{2}, \gamma\right]$, then $K_{i} \in \mathbf{B}$ and $K_{i+1} \in \mathbf{A}$.

Let $g: \mathbb{S} \rightarrow \mathbb{S}$ be the golden rigid rotation with rotation number $\gamma$. The map $g$ has the property that

$$
g \circ \pi_{\mathbb{S}}(x)=\pi_{\mathbb{S}}(x+1),
$$

for every $x \in \mathbb{R}$. Since $G_{A}: \mathbb{T} \rightarrow \mathbb{T}$ is an Anosov automorphism, we obtain that $g\left(z_{i}\right)=z_{i+1}$, for every $i \in \mathbb{N}_{0}$. Let us denote by $\ell\left(y_{0}, y_{i}\right)$ the leaf segment with endpoints $y_{0}$ and $y_{i}$. Since $G_{A}: \mathbb{T} \rightarrow \mathbb{T}$ is the golden Anosov automorphism, if the leaf $\ell\left(y_{0}, y_{i}\right)$ contains $m_{A}$ spanning leaf segments of the Markov rectangle $A$ and $m_{B}$ spanning leaf segments of the Markov rectangle $B$, then $G_{A}\left(\ell\left(y_{0}, y_{i}\right)\right)=$ $\ell\left(y_{0}, G_{A}\left(y_{i}\right)\right)$ contains $m_{A}+m_{B}$ spanning leaf segments of the Markov rectangle $A$ and $m_{A}$ spanning leaf segments of the Markov rectangle $B$. Hence, by induction, we have that $G_{A}\left(y_{F_{i}}\right)=y_{F_{i+1}}$, where $F_{1}, F_{2}, \ldots$ are the Fibonacci numbers. Thus, for every $i \in \mathbb{N}$, we have that $G_{A}^{i-1}\left(y_{1}\right)=y_{F_{i}}$, and so $d\left(y_{0}, y_{F_{i}}\right)=\gamma^{i}$ and $\pi\left((-\gamma)^{i}, 0\right)=$ $y_{F_{i}}$. Thus $g^{F_{i}}\left(z_{0}\right)=z_{F_{i}}=\pi_{\mathbb{S}}\left((-\gamma)^{i}\right)$. Since $g$ is the golden rigid rotation, we have that

$$
g^{F_{i}}\left(\pi_{\mathbb{S}}(x)\right)=\pi_{\mathbb{S}}\left(x+(-\gamma)^{i}\right),
$$

for every $x \in \mathbb{R}$ and $i \in \mathbb{N}$. Hence, for every $i \in \mathbb{N}$ with Fibonacci decomposition $F_{n_{0}}, \ldots, F_{n_{p}}$, we obtain

$$
z_{i}=g^{i}\left(z_{0}\right)=g^{F_{n_{0}}+\cdots+F_{n_{p}}}\left(z_{0}\right) .
$$

Thus, by equality (3.1), we have that

$$
g^{F_{n_{0}}+\cdots+F_{n_{p}}}\left(z_{0}\right)=\pi_{\mathbb{S}}\left(\sum_{i=0}^{p}(-\gamma)^{n_{i}}\right) .
$$

Noting that $\sum_{i=0}^{+\infty} \gamma^{2 i}=\left(1-\gamma^{2}\right)^{-1}=\gamma^{-1}$, we obtain

$$
\sum_{i=0}^{p}(-\gamma)^{n_{i}}<\sum_{j \geq 0} \gamma^{2+2 j}=\gamma
$$

and

$$
\sum_{i=0}^{p}(-\gamma)^{n_{i}}>\sum_{j \geq 0}-\gamma^{1+2 j}=-1 .
$$

Therefore, taking $w_{i}=\sum_{j=0}^{p}(-\gamma)^{n_{j}} \in[-1, \gamma]$ we obtain that $\pi_{\mathbb{S}}\left(w_{i}\right)=z_{i}$. Now, there are six distinct cases to consider depending upon the Fibonacci decomposition $F_{n_{0}}, \ldots, F_{n_{p}}$ of $i$ (see Figure 6):

(i) if $n_{p}=1$ and $n_{p-1}$ is odd, then $w_{i} \in[-1,-\gamma)$, and so $K_{i} \in \mathbf{B}$ and $K_{i+1} \in \mathbf{A}$;

(ii) if $n_{p}=1$ and $n_{p-1}$ is even, then $w_{i} \in\left(-\gamma,-\gamma^{2}\right)$, and so $K_{i} \in \mathbf{A}$ and $K_{i+1} \in \mathbf{B}$

(iii) if $n_{p}=2$ and $n_{p-1}$ is odd, then $w_{i} \in\left(\gamma^{3}, \gamma^{2}\right)$, and so $K_{i}, K_{i+1} \in \mathbf{A}$;

(iv) if $n_{p}=2$ and $n_{p-1}$ is even, then $w_{i} \in\left(\gamma^{2}, \gamma\right]$, and so $K_{i} \in \mathbf{B}$ and $K_{i+1} \in \mathbf{A}$; 


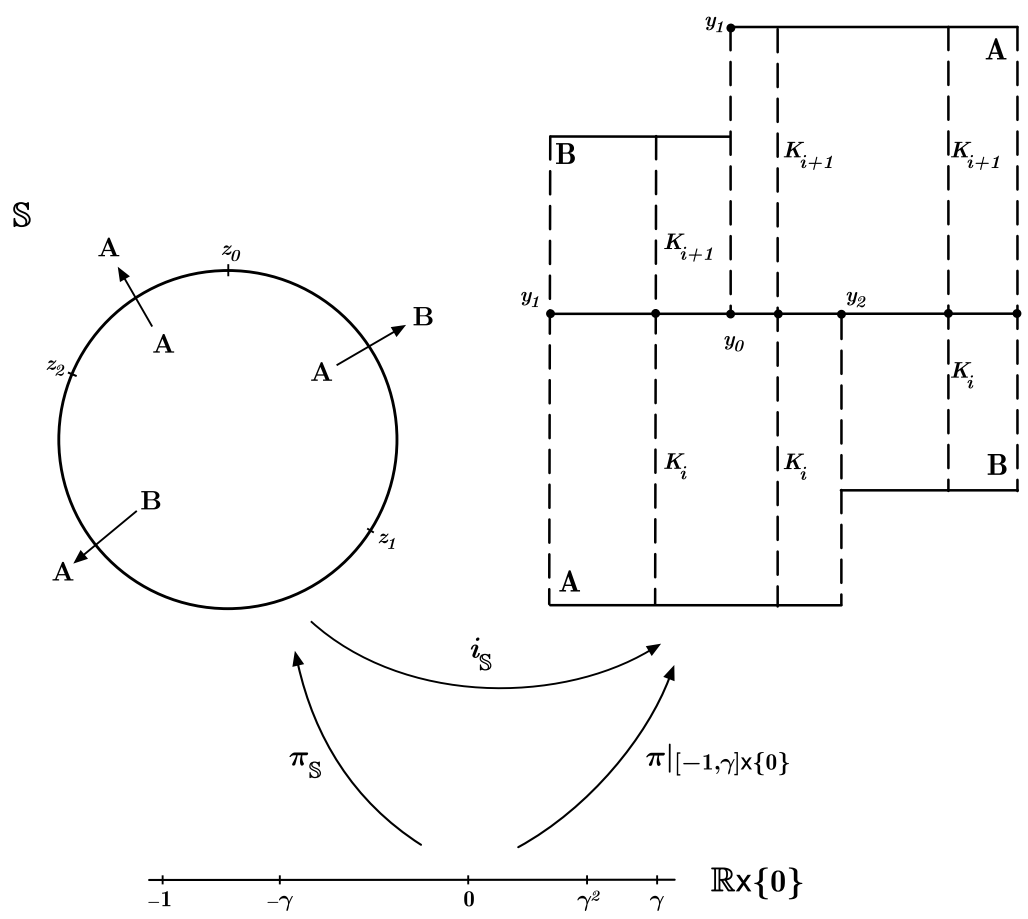

FiguRE 5 . The map $i_{\mathbb{S}} \circ \pi_{\mathbb{S}}$.

(v) if $n_{p}>2$ and $n_{p}$ is odd, then $w_{i} \in\left(-\gamma^{2}, 0\right)$, and so $K_{i} \in \mathbf{A}$ and $K_{i+1} \in \mathbf{B}$; (vi) if $n_{p}>2$ and $n_{p}$ is even, then $w_{i} \in\left(0, \gamma^{3}\right)$, and so $K_{i}, K_{i+1} \in \mathbf{A}$.

3.3. Fibonacci shift. We define the Fibonacci shift $\sigma: \mathbb{N} \rightarrow \mathbb{N}$ as follows. For every $i \in \mathbb{N}$, let $F_{n_{0}}, \ldots, F_{n_{p}}$ be the Fibonacci decomposition associated to $i$, i.e. $i=F_{n_{0}}+\cdots+F_{n_{p}}$. If $n_{p} \neq 1$, then we define $\sigma(i)=F_{n_{0}+1}+\cdots+F_{n_{p}+1}$. If $n_{p}=1$ and $n_{p-1}$ is odd, then we define $\sigma(i)=F_{n_{0}+1}+\cdots+F_{n_{p-1}+1}+F_{1}$. If $n_{p}=1$ and $n_{p-1}$ is even, then we define $\sigma(i)=F_{n_{0}+1}+\cdots+F_{n_{p-1}+1}+F_{2}$. Hence, the inverse of the Fibonacci shift $\sigma^{-1}(i)$ is given as follows: if $n_{p} \neq 1$, then $\sigma^{-1}(i)=F_{n_{0}-1}+\cdots+F_{n_{p}-1}$; if $n_{p}=1$ and $n_{p-1}$ is even, then $\sigma^{-1}(i)=$ $F_{n_{0}-1}+\cdots+F_{n_{p-1}-1}+F_{1}$; if $n_{p}=1$ and $n_{p-1}$ is odd, then $\sigma^{-1}(i)=\emptyset$.

Remark 3.3. We observe that for $F_{n_{p}}=F_{1}$ the definition of the Fibonacci shift is somewhat unnatural. This is due to the fact that we consider, for simplicity, the Fibonacci sequence $F_{1}=1, F_{2}=2, F_{3}=3, F_{4}=5, \ldots$ instead of the sequence $F_{0}=1, F_{1}=1, F_{2}=2, F_{3}=3, F_{4}=5, \ldots$ If we consider the sequence $F_{0}=$ $1, F_{1}=1, F_{2}=2, F_{3}=3, F_{4}=5, \ldots$, then we have to change the Fibonacci decomposition of the number $i$ accordingly with the following rule: if $n_{p-1}$ is odd and $i-\left(F_{n_{0}}+\cdots+F_{n_{p-1}}\right)=1$, then $n_{p}=0$. In this case, for every $i \in \mathbb{N}$, we define $\sigma(i)=F_{n_{0}+1}+\cdots+F_{n_{p-1}+1}+F_{n_{p}+1}$. We get that if $n_{p} \neq 0$, then $\sigma^{-1}(i)=F_{n_{0}-1}+\cdots+F_{n_{p}-1}$, and if $n_{p}=0$, then $\sigma^{-1}(i)=\emptyset$. We claim that for this new Fibonacci decomposition all the statements in the paper will hold with the corresponding simple alterations. 


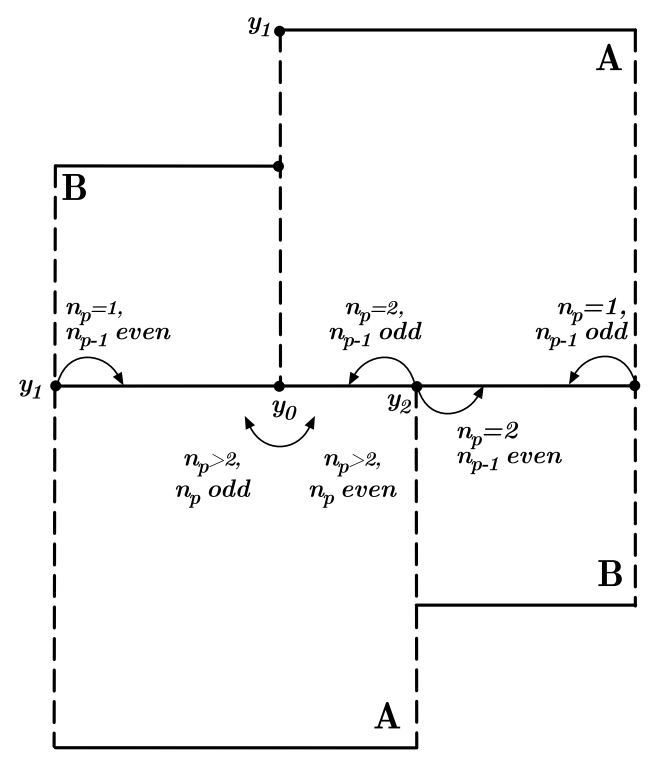

Figure 6 . The location of the point $y_{i}$ depending upon the Fibonacci decomposition of $i$.

3.4. Matching condition. We say that a sequence $\left(a_{i}\right)_{i \in \mathbb{N}}$ satisfies the matching condition if, for every $i=F_{n_{0}}+\cdots+F_{n_{p}}$, the following conditions hold:

(i) If either $\left(n_{p}=1\right.$ and $n_{p-1}$ is odd) or ( $n_{p}=2$ and $n_{p-1}$ is even), then

$$
a_{\sigma(i)}=a_{i}\left(a_{\sigma(i)+1}+1\right)^{-1} \text {. }
$$

(ii) If either ( $n_{p}=1$ and $n_{p-1}$ is even) or ( $n_{p}>2$ and $n_{p}$ is odd), then

$$
a_{\sigma(i)}=a_{i}\left(a_{\sigma(i)-1}^{-1}+1\right) \text {. }
$$

(iii) If either $\left(n_{p}=2\right.$ and $n_{p-1}$ odd) or ( $n_{p}>2$ and $n_{p}$ is even), then

$$
a_{\sigma(i)}=\frac{a_{i}\left(1+a_{\sigma(i)-1}\right)}{a_{\sigma(i)-1}\left(1+a_{\sigma(i)+1}\right)} .
$$

Lemma 3.4. The sequence $\left(a_{i}^{G}\right)_{i \in \mathbb{N}}$ satisfies the matching condition.

Proof. By Lemma 3.1, we have that $a_{i}^{G}=\sigma_{G}\left(K_{i}: K_{i+1}\right)$, for every $i \in \mathbb{N}$. Hence Lemma 3.4 follows from putting together Lemma 2.2 and Lemma 3.2 .

Remark 3.5. Every sequence $\left(b_{i}\right)_{i \in \mathbb{N} \backslash \sigma(\mathbb{N})}$ determines, uniquely, a sequence $\left(a_{i}\right)_{i \in \mathbb{N}}$ satisfying the matching condition as follows: for every $i \in \mathbb{N} \backslash \sigma(\mathbb{N})$, define $a_{i}=b_{i}$ and, for every $i \in \sigma(\mathbb{N})$, define $a_{\sigma(i)}$ using the matching condition and the elements 


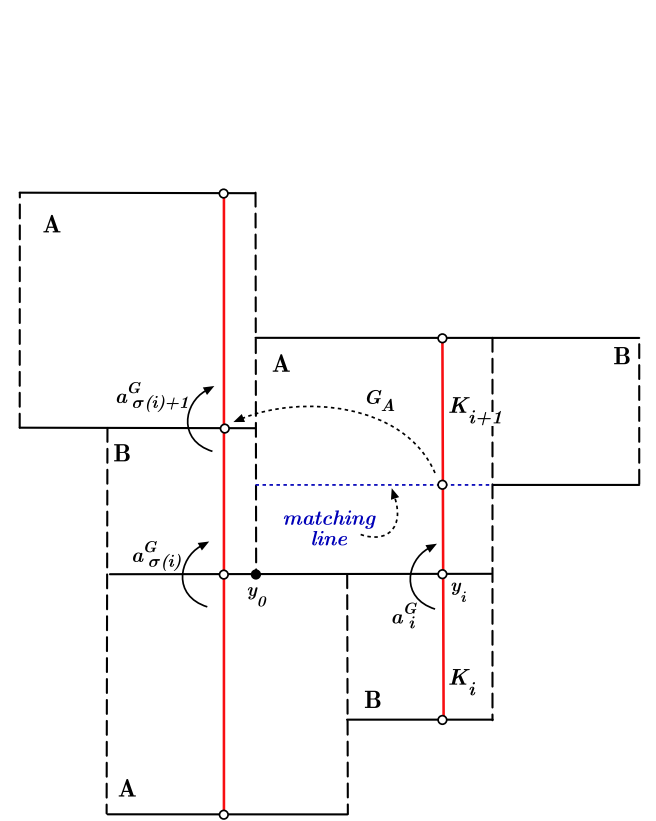

(A) Condition (i)

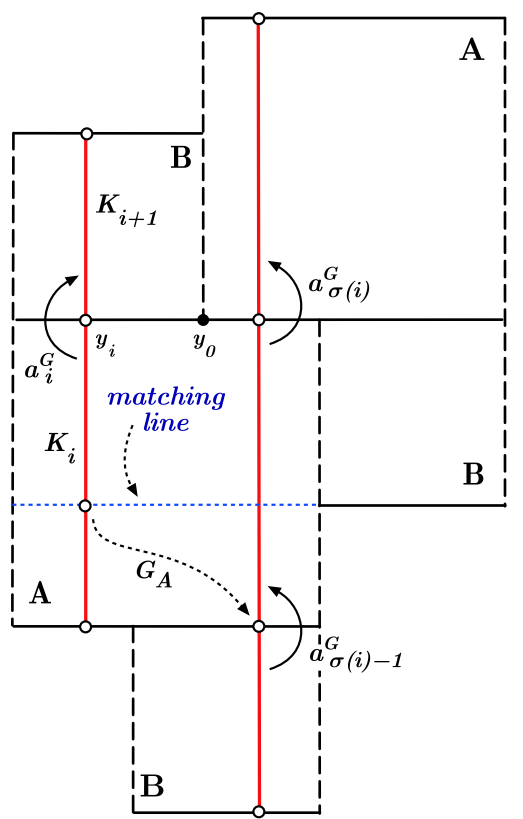

(B) Condition (ii)

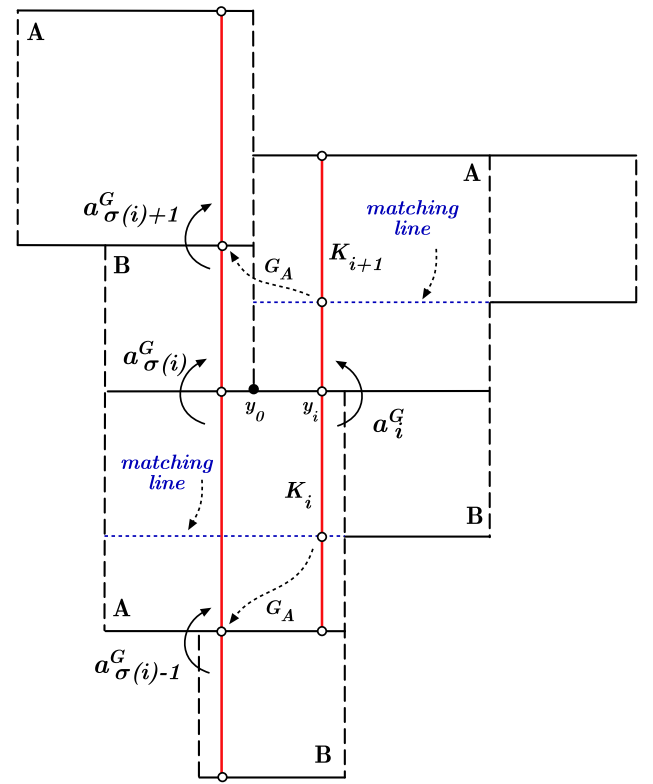

(c) Condition (iii)

FIgURE 7. The matching condition.

$a_{j}$ of the sequence with

$$
j \in\{j: 2 \leq j<\sigma(i) \vee j \in \mathbb{N} \backslash \sigma(\mathbb{N})\} .
$$




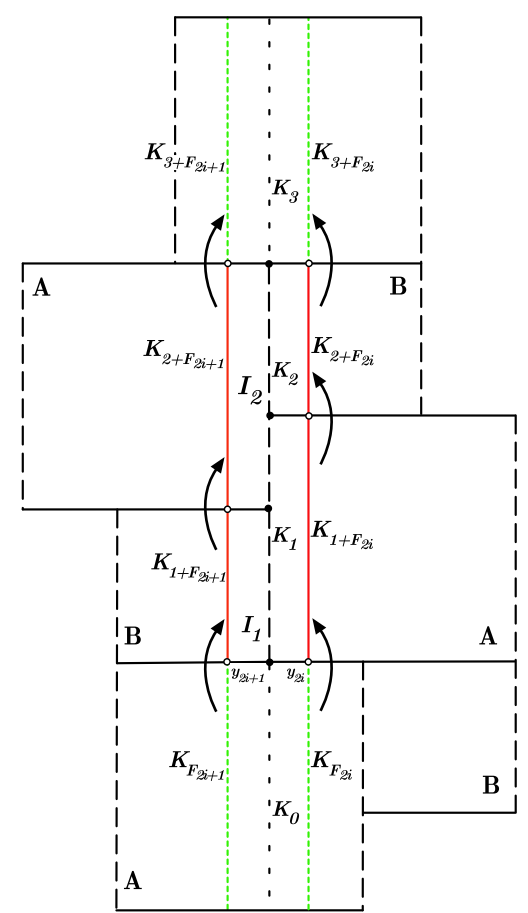

FiguRE 8. The boundary condition.

3.5. Boundary condition. A sequence $\left(a_{i}\right)_{i \in \mathbb{N}}$ satisfies the boundary condition if the following limits are well defined and satisfy the inequalities:
(i) $\lim _{i \rightarrow+\infty} a_{F_{i}+2}^{-1}\left(1+a_{F_{i}+1}^{-1}\right) \neq 0$.
(ii) $\lim _{i \rightarrow+\infty} a_{F_{i}}\left(1+a_{F_{i}+1}\right) \neq 0$.

Lemma 3.6. The sequence $\left(a_{i}^{G}\right)_{i \in \mathbb{N}}$ satisfies the boundary condition.

Proof. We observe that $d\left(K_{F_{n}}, K_{0}\right)=\gamma^{n}, d\left(K_{F_{2 n+1}+1}, I_{1}\right)=\gamma^{2 n+1}, d\left(K_{F_{2 n+1}+2}, I_{2}\right)$ $=\gamma^{2 n+1}, d\left(K_{F_{2 n}+1}, K_{1}\right)=\gamma^{2 n}, d\left(K_{F_{2 n}+2}, K_{2}\right)=\gamma^{2 n}$ and $d\left(K_{F_{n}+3}, K_{3}\right)=\gamma^{n}$ (see Figure (8). By continuity of $\sigma_{a}$, we have that

$$
\begin{aligned}
\lim _{n \rightarrow \infty} a_{F_{2 n}}^{G} & \left(1+a_{F_{2 n}+1}^{G}\right) \\
= & \lim _{n \rightarrow \infty} \sigma_{G}\left(K_{F_{2 n}}: K_{F_{2 n}+1}\right)\left(1+\sigma_{G}\left(K_{F_{2 n}+1}: K_{F_{2 n}+2}\right)\right) \\
& =\sigma_{G}\left(K_{0}: K_{1}\right)\left(1+\sigma_{G}\left(K_{1}: K_{2}\right)\right)
\end{aligned}
$$

and

$$
\begin{aligned}
\lim _{n \rightarrow \infty} a_{F_{2 n+1}}^{G}\left(1+a_{F_{2 n+1}+1}^{G}\right) & \\
= & \lim _{n \rightarrow \infty} \sigma_{G}\left(K_{F_{2 n+1}}: K_{F_{2 n+1}+1}\right)\left(1+\sigma_{G}\left(K_{F_{2 n+1}+1}: K_{F_{2 n+1}+2}\right)\right) \\
& =\sigma_{G}\left(K_{0}: I_{1}\right)\left(1+\sigma_{G}\left(I_{1}: I_{2}\right)\right) .
\end{aligned}
$$


Hence, by equality (2.9), we obtain that the golden sequence $\left(a_{i}^{G}\right)_{i \in \mathbb{N}}$ satisfies the boundary condition (i). By continuity of $\sigma_{G}$, we have that

$$
\begin{aligned}
\lim _{n \rightarrow \infty}\left(a_{F_{2 n}+2}^{G}\right)^{-1}\left(1+\left(a_{F_{2 n}+1}^{G}\right)^{-1}\right) & \\
& =\lim _{n \rightarrow \infty} \sigma_{G}\left(K_{F_{2 n}+3}: K_{F_{2 n}+2}\right)\left(1+\sigma_{G}\left(K_{F_{2 n}+2}: K_{F_{2 n}+1}\right)\right) \\
& =\sigma_{G}\left(K_{3}: K_{2}\right)\left(1+\sigma_{G}\left(K_{2}: K_{1}\right)\right)
\end{aligned}
$$

and

$$
\begin{aligned}
\lim _{n \rightarrow \infty}\left(a_{F_{2 n+1}+2}^{G}\right)^{-1} & \left(1+\left(a_{F_{2 n+1}+1}^{G}\right)^{-1}\right) \\
& =\lim _{n \rightarrow \infty} \sigma_{G}\left(K_{F_{2 n+1}+3}: K_{F_{2 n+1}+2}\right)\left(1+\sigma_{G}\left(K_{F_{2 n+1}+2}: K_{F_{2 n+1}+1}\right)\right) \\
& =\sigma_{G}\left(K_{3}: I_{2}\right)\left(1+\sigma_{G}\left(I_{2}: I_{1}\right)\right) .
\end{aligned}
$$

Hence, by equality (2.10) we obtain that the golden sequence $\left(a_{i}^{G}\right)_{i \in \mathbb{N}}$ satisfies the boundary condition (ii).

3.6. The exponentially fast Fibonacci repetitive property. A sequence $\left(a_{i}\right)_{i \in \mathbb{N}}$ is said to be exponentially fast Fibonacci repetitive if there exist constants $C \geq 0$ and $0<\mu<1$ such that

$$
\left|a_{i+F_{m}}-a_{i}\right| \leq C \mu^{m}
$$

for every $m \geq 5$ and $3 \leq i<F_{m-1}$ and, also, for $i \in\{1,2\}$ if $m$ is even.

Lemma 3.7. The sequence $\left(a_{i}^{G}\right)$ satisfies the exponentially fast Fibonacci repetitive property.

Proof. For every $m \geq 5$, we have that either $m=2 n$ or $m=2 n+1$ for some $n \geq 2$ (see Figure 9). Recall that $K_{i} \cap K_{i+1}=\left\{y_{i}\right\}$, for every $i \in \mathbb{N}_{0}$.

(i) Case $m=2 n$. For $1 \leq i<F_{2 n-1}$, the unstable spanning leaf segments $K_{i}$, $K_{i+1}, K_{i+F_{2 n}}$ and $K_{i+1+F_{2 n}}$ belong to $G^{2 n}(\mathbf{A})$. Hence, we obtain that

$$
d\left(K_{i}, K_{i+F_{2 n}}\right) \leq C_{0}\left|y_{i}-y_{i+F_{2 n}}\right| \leq C_{0} \gamma^{2 n},
$$

for some $C_{0} \geq 1$ and $0<\gamma<1$. By Hölder continuity of the solenoid function, there exist constants $C \geq 1$ and $\alpha<1$ such that

$$
\begin{aligned}
\left|a_{i}^{G}-a_{i+F_{2 n}}^{G}\right| & =\left|\sigma_{G}\left(K_{i}: K_{i+1}\right)-\sigma_{G}\left(K_{i+F_{2 n}}: K_{i+1+F_{2 n}}\right)\right| \\
& <C\left(\gamma^{2 n}\right)^{\alpha} \\
& =C\left(\gamma^{\alpha}\right)^{2 n} .
\end{aligned}
$$

(ii) Case $m=2 n+1$. For $3 \leq i<F_{2 n}$, the unstable spanning leaf segments $K_{i}$, $K_{i+1}, K_{i+F_{2 n+1}}$ and $K_{i+1+F_{2 n+1}}$ belong to $G^{2 n}(\mathbf{B})$. Hence, we obtain that

$$
d\left(K_{i}, K_{i+F_{2 n+1}}\right) \leq C_{0}\left|y_{i}-y_{i+F_{2 n+1}}\right| \leq C_{0} \gamma^{2 n+1},
$$

for some $C_{0} \geq 1$ and $0<\gamma<1$. By Hölder continuity of the solenoid function, there exist constants $C \geq 1$ and $\alpha<1$ such that

$$
\begin{aligned}
\left|a_{i}^{G}-a_{i+F_{2 n+1}}^{G}\right| & =\left|\sigma_{G}\left(K_{i}: K_{i+1}\right)-\sigma_{G}\left(K_{i+F_{2 n+1}}: K_{i+1+F_{2 n+1}}\right)\right| \\
& <C\left(\gamma^{2 n+1}\right)^{\alpha} \\
& =C \gamma^{\alpha}\left(\gamma^{\alpha}\right)^{2 n} .
\end{aligned}
$$

Hence, the sequence $\left(a_{i}^{G}\right)$ satisfies the exponentially fast Fibonacci repetitive property with $\mu=\gamma^{\alpha}$. 


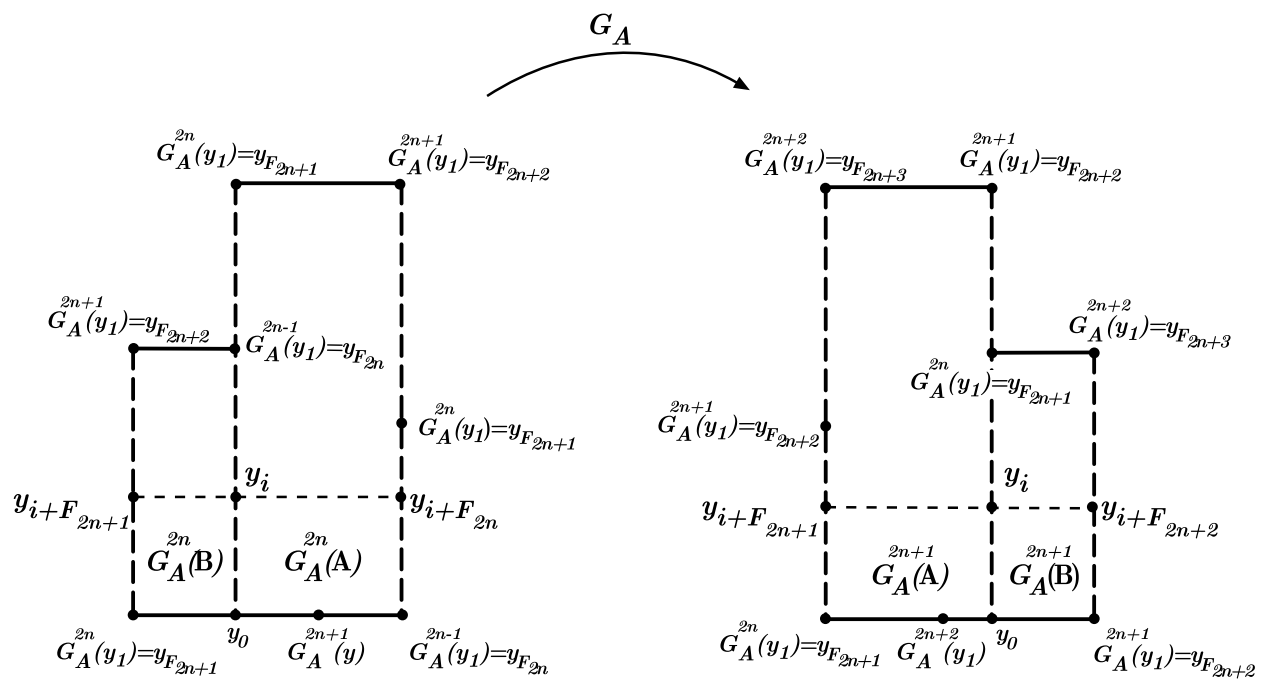

Figure 9. The exponentially fast Fibonacci repetitive condition.

3.7. Golden tilings. A tiling $\mathcal{T}=\left\{I_{i} \subset \mathbb{R}: i \in \mathbb{N}\right\}$ of the positive real line is a collection of tiling intervals $I_{i}$, with the following properties:

(i) the tiling intervals are closed intervals;

(ii) any two distinct intervals have disjoint interiors;

(iii) the union $\bigcup_{i \in \mathbb{N}} I_{i}$ is equal to the positive real line;

(iv) for every $i \in \mathbb{N}$ the intersection of the tiling intervals $I_{i}$ and $I_{i+1}$ is only a point, which is an endpoint, simultaneously, of both intervals.

The tilings $\mathcal{T}_{1}=\left\{I_{i} \subset \mathbb{R}: i \in \mathbb{N}\right\}$ and $\mathcal{T}_{2}=\left\{J_{i} \subset \mathbb{R}: i \in \mathbb{N}\right\}$ of the positive real line are in the same affine class if there exists an affine map $h: \mathbb{R} \rightarrow \mathbb{R}$ such that $h\left(I_{i}\right)=J_{i}$, for every $i \in \mathbb{N}$. Thus, every positive sequence $\left(a_{i}\right)_{i \in \mathbb{N}}$ determines a unique affine class of tilings $\mathcal{T}=\left\{I_{i} \subset \mathbb{R}: i \in \mathbb{N}\right\}$ such that $a_{i}=\left|I_{i+1}\right| /\left|I_{i}\right|$, and vice versa.

Definition 3.8. A golden sequence $\left(a_{i}\right)_{i \in \mathbb{N}}$ is an exponentially fast Fibonacci repetitive sequence that satisfies the matching and the boundary conditions. A tiling $\mathcal{T}=\left\{I_{i} \subset \mathbb{R}: i \in \mathbb{N}\right\}$ of the positive real line is golden if the corresponding sequence $\left(a_{i}=\left|I_{i+1}\right| /\left|I_{i}\right|\right)_{i \in \mathbb{N}}$ is a golden sequence.

We say that a golden tiling $\mathcal{T}_{R}$ is rigid if its associated golden sequence is rigid (see Definition 1.2).

3.8. Proof of Theorem 1.1, By Lemma 3.1, the map $G \rightarrow\left(a_{i}^{G}\right)_{i \in \mathbb{N}}$ determines a correspondence between Anosov diffeomorphisms $G$ in $\mathcal{G}$ and golden sequences such that $a_{i}^{G}=\sigma_{G}\left(K_{i}: K_{i+1}\right)$. Putting together Lemma 3.4 Lemma 3.6 and Lemma 3.7, we get that $\left(a_{i}^{G}\right)_{i \in \mathbb{N}}$ is a golden sequence. By Corollary 2.6, any two $C^{1+}$ Anosov diffeomorphisms, $G_{1}$ and $G_{2}$, that are $C^{1+}$ smooth conjugate determine the same solenoid functions $\sigma_{G_{1}}=\sigma_{G_{2}}$. Hence, by Lemma 3.1, $\left(a_{i}^{G_{1}}\right)_{i \in \mathbb{N}}=\left(a_{i}^{G_{2}}\right)_{i \in \mathbb{N}}$.

Conversely, given a golden sequence $\left(a_{i}\right)_{i \in \mathbb{N}}$ we construct a solenoid function $\sigma_{a}$ in sol as follows. Recall that $\mathbb{L}=\left\{\left(K_{i}: K_{i+1}\right), i \in \mathbb{N}\right\}$ is a dense set in sol. We define $\sigma_{a}\left(K_{i}: K_{i+1}\right)=a_{i}$, for every $\left(K_{i}: K_{i+1}\right) \in \mathbb{L}$. Since the sequence 
$\left(a_{i}\right)_{i \in \mathbb{N}}$ is exponentially fast Fibonacci repetitive, similar to the proof of Lemma 3.7 we get that $\sigma_{a} \mid \mathbb{L}$ is Hölder continuous. Hence, using the fact that $\mathbb{L}$ is dense in sol, we define $\sigma_{a}$ in sol as the unique Hölder continuous extension of $\sigma_{a} \mid \mathbb{L}$ to sol. Now, it is enough to check that the Hölder continuous function $\sigma_{a}$ in sol satisfies the matching and the boundary condition. Since $\left(a_{i}\right)_{i \in \mathbb{N}}$ satisfies the golden matching condition, similar to the proof of Lemma 3.4, we have that $\sigma_{a} \mid \mathbb{L}$ satisfies the matching condition in $\mathbb{L}$. Hence, using the fact that $\sigma_{a}$ in sol is a continuous function, we get that the $\sigma_{a}$ in sol also satisfies the matching condition. Recall, from Section 1.1, the definition of $K_{0}, K_{1}, K_{2}$ and $K_{3}$. Recall that the spanning leaf segments $I_{1}$ and $I_{2}$ are, respectively, the right boundaries of the Markov rectangles $B$ and $A$, as in Section 2.9. We observe that $d\left(K_{F_{n}}, K_{0}\right)=\gamma^{n}, d\left(K_{F_{2 n+1}+1}, I_{1}\right)=$ $\gamma^{2 n+1}, d\left(K_{F_{2 n+1}+2}, I_{2}\right)=\gamma^{2 n+1}, d\left(K_{F_{2 n}+1}, K_{1}\right)=\gamma^{2 n}, d\left(K_{F_{2 n}+2}, K_{2}\right)=\gamma^{2 n}$ and $d\left(K_{F_{n}+3}, K_{3}\right)=\gamma^{n}$. By continuity of $\sigma_{a}$, we have that

$$
\begin{aligned}
\sigma_{a}\left(K_{0}: K_{1}\right) & \left(1+\sigma_{a}\left(K_{1}: K_{2}\right)\right) \\
= & \lim _{n \rightarrow \infty} \sigma_{a}\left(K_{F_{2 n}}: K_{F_{2 n}+1}\right)\left(1+\sigma_{a}\left(K_{F_{2 n}+1}: K_{F_{2 n}+2}\right)\right) \\
= & \lim _{n \rightarrow \infty} a_{F_{2 n}}\left(1+a_{F_{2 n}+1}\right)
\end{aligned}
$$

and

$$
\begin{aligned}
\sigma_{a}\left(K_{0}: I_{1}\right) & \left(1+\sigma_{a}\left(I_{1}: I_{2}\right)\right) \\
& =\lim _{n \rightarrow \infty} \sigma_{a}\left(K_{F_{2 n+1}}: K_{F_{2 n+1}+1}\right)\left(1+\sigma_{a}\left(K_{F_{2 n+1}+1}: K_{F_{2 n+1}+2}\right)\right) \\
& =\lim _{n \rightarrow \infty} a_{F_{2 n+1}}\left(1+a_{F_{2 n+1}+1}\right) .
\end{aligned}
$$

Hence, by the boundary condition (i) of the golden sequence $\left(a_{i}\right)_{i \in \mathbb{N}}$, we obtain that $\sigma_{a}$ satisfies equality (2.9). By continuity of $\sigma_{a}$, we have that

$$
\begin{aligned}
\sigma_{a}\left(K_{3}: K_{2}\right) & \left(1+\sigma_{a}\left(K_{2}: K_{1}\right)\right) \\
& =\lim _{n \rightarrow \infty} \sigma_{a}\left(K_{F_{2 n}+3}: K_{F_{2 n}+2}\right)\left(1+\sigma_{a}\left(K_{F_{2 n}+2}: K_{F_{2 n}+1}\right)\right) \\
& =\lim _{n \rightarrow \infty} a_{F_{2 n}+2}^{-1}\left(1+a_{F_{2 n}+1}^{-1}\right)
\end{aligned}
$$

and

$$
\begin{aligned}
\sigma_{a}\left(K_{3}: I_{2}\right) & \left(1+\sigma_{a}\left(I_{2}: I_{1}\right)\right) \\
& =\lim _{n \rightarrow \infty} \sigma_{a}\left(K_{F_{2 n+1}+3}: K_{F_{2 n+1}+2}\right)\left(1+\sigma_{a}\left(K_{F_{2 n+1}+2}: K_{F_{2 n+1}+1}\right)\right) \\
& =\lim _{n \rightarrow \infty} a_{F_{2 n+1}+2}^{-1}\left(1+a_{F_{2 n+1}+1}^{-1}\right) .
\end{aligned}
$$

Hence, by the boundary condition (ii) of the golden sequence $\left(a_{i}\right)_{i \in \mathbb{N}}$, we obtain that $\sigma_{a}$ satisfies equality (2.10). Therefore, $\sigma_{a}$ is a solenoid function.

\section{Complete set of holonomies}

Let $h_{G}: \mathbb{T} \rightarrow \mathbb{T}$ be the topological conjugacy between the Anosov automorphism $G_{A}$ and $G$. The rectangles $h_{G}(\mathbf{A})$ and $h_{G}(\mathbf{B})$ form a Markov partition $\mathcal{M}_{G}$ of $G$. Suppose that $M$ and $N$ are Markov rectangles and that $x \in \operatorname{int}(M)$ and $y \in \operatorname{int}(N)$. We say that $x$ and $y$ are stable holonomically related if (i) there is an stable leaf segment $\ell^{u}(x, y)$ such that $\partial \ell^{u}(x, y)=\{x, y\}$, and (ii) $\ell^{u}(x, y) \subset$ $\ell^{u}(x, M) \cup \ell^{u}(y, N)$. Let $P=P_{\mathcal{M}}$ be the set of all pairs $(M, N)$ such that there are points $x \in \operatorname{int}(M)$ and $y \in \operatorname{int}(N)$ unstable holonomically related. 
For every Markov rectangle $M \in \mathcal{M}_{G}$, choose an unstable spanning leaf segment $\ell(x, M)$ in $M$ for some $x \in M$. Let $\mathcal{I}=\left\{\ell_{M}: M \in \mathcal{M}\right\}$. For every pair $(M, N) \in P$, there are maximal leaf segments $\ell_{(M, N)}^{D} \subset \ell_{M}, \ell_{(M, N)}^{C} \subset \ell_{N}$ such that the unstable holonomy $h_{(M, N)}: \ell_{(M, N)}^{D} \rightarrow \ell_{(M, N)}^{C}$ is well defined. We call such holonomies $h_{(M, N)}: \ell_{(M, N)}^{D} \rightarrow \ell_{(M, N)}^{C}$ the unstable primitive holonomies associated to the Markov partition $\mathcal{M}_{G}$. The complete set of unstable holonomies $\mathcal{H}_{G}$ consists of all stable primitive holonomies and their inverses. In Figure 10, we exhibit the complete set of unstable holonomies

$$
\mathcal{H}_{G}=\left\{h_{(\mathbf{A}, \mathbf{A})}, h_{(\mathbf{A}, \mathbf{A})}^{-1}, h_{(\mathbf{A}, \mathbf{B})}, h_{(\mathbf{A}, \mathbf{B})}^{-1}, h_{(\mathbf{B}, \mathbf{A})}, h_{(\mathbf{B}, \mathbf{A})}^{-1}\right\}
$$

associated to the Markov partition $\mathcal{M}_{G}$.

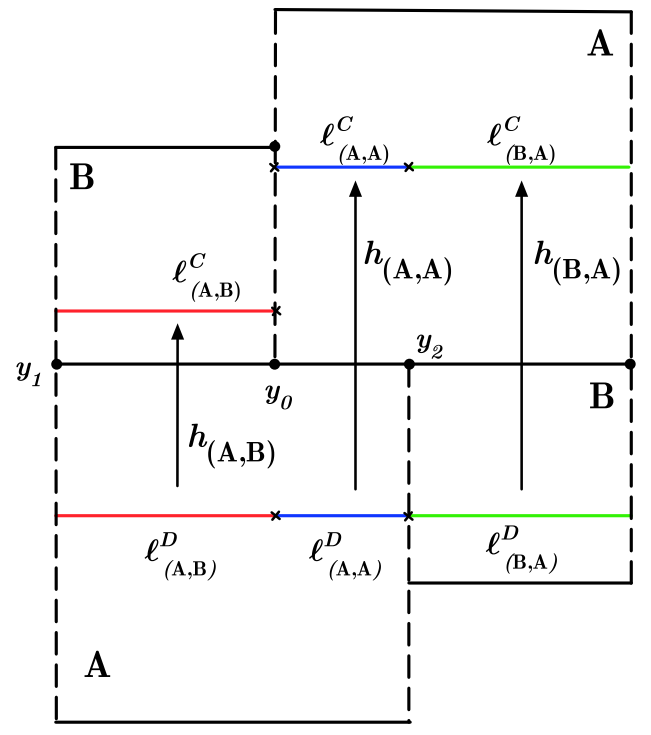

Figure 10. A complete set of unstable holonomies $\mathcal{H}_{G}$ associated to the Markov partition $\mathcal{M}_{G}$.

A diffeomorphism $\theta: I \rightarrow J$ is said to be $C^{1+z y g m u n d}$ if $\theta$ is $C^{1}$ and the derivative $\theta^{\prime}$ satisfies the zygmund condition, i.e. for all points $x, y \in I$,

$$
\left|\theta^{\prime}(x)+\theta^{\prime}(y)-2 \theta^{\prime}\left(\frac{x+y}{2}\right)\right|=\chi_{\theta}(|y-x|),
$$

where the function $\chi_{\theta}$ is such that $\chi_{\theta}(t) \rightarrow 0$ when $t \rightarrow 0$. In particular, a $C^{2+\beta}$ diffeomorphism, with $\beta>0$, is a $C^{1+z y g m u n d}$ diffeomorphism. The importance of this smooth class follows from the fact that it corresponds to maps that distort cross-ratios of quadruples of points in $I$ by an amount that is $o(|I|)$ (see [7, [18] and [28]).

Definition 4.1. A complete set of unstable holonomies $\mathcal{H}_{G}$ is $C^{1+\text { zygmund }}$ if all holonomies in $\mathcal{H}_{G}$ are $C^{1+\text { zygmund }}$ with respect to the atlas $\mathcal{L}^{s}(G, \rho)$. 
4.1. Proof of Theorem 1.3. By Lemma 3.3 and Theorem 4.1 in 22, if $G$ has a $C^{1+\text { zygmund }}$ complete system of unstable holonomies, then $\sigma_{G}=\sigma_{G_{A}}$. By Lemma 3.2. for $i \in \mathbb{N}$ with Fibonacci decomposition $F_{n_{0}}, \ldots, F_{n_{p}}$, we have that the following conditions hold:

(i) If either $\left(n_{p}=1\right.$ and $n_{p-1}$ is odd) or $\left(n_{p}=2\right.$ and $n_{p-1}$ is even), then $K_{i} \in \mathbf{B}$ and $K_{i+1} \in \mathbf{A}$. Hence, $a_{i}=\sigma_{G_{A}}\left(K_{i}: K_{i+1}\right)=\gamma^{-1}$.

(ii) If either ( $n_{p}=1$ and $n_{p-1}$ is even) or $\left(n_{p}>2\right.$ and $n_{p}$ is odd), then $K_{i} \in \mathbf{A}$ and $K_{i+1} \in \mathbf{B}$. Hence, $a_{i}=\sigma_{G_{A}}\left(K_{i}: K_{i+1}\right)=\gamma$.

(iii) If either $\left(n_{p}=2\right.$ and $n_{p-1}$ is odd) or $\left(n_{p}>2\right.$ and $n_{p}$ is even), then $K_{i}, K_{i+1} \in \mathbf{A}$. Hence, $a_{i}=\sigma_{G_{A}}\left(K_{i}: K_{i+1}\right)=1$.

Thus, from conditions (i), (ii) and (iii) we conclude that $\left(a_{i}^{G}\right)_{i \in \mathbb{N}}$ is the rigid golden sequence.

\section{ACKNOWLEDGMENTS}

We are grateful to Alby Fisher, David Rand, Dennis Sullivan, Flávio Ferreira and Yunping Jiang for a number of very fruitful and useful discussions on this work and for their friendship and encouragement. We thank the LIAAD-INESC Porto LA, Calouste Gulbenkian Foundation, PRODYN-ESF, POCTI and POSI by FCT and the Ministério da Ciência e da Tecnologia and the Pluriannual Funding Program of LIAAD-INESC Porto LA and of Research Centre of Mathematics of the University of Minho for their financial support. Part of this research was started during a visit by the authors to the IHES and CUNY, and continued at the Isaac Newton Institute, the University of Warwick, IMPA, MSRI and SUNY. We thank them for their hospitality.

\section{REFERENCES}

[1] Anosov, D.V., Tangent fields of transversal foliations in U-systems, Math. Notes Acad. Sci., USSR, 2 (5) (1967). MR0242190 (39:3523)

[2] Bedford T., Fisher, A.M., Ratio geometry, rigidity and the scenery process for hyperbolic Cantor sets, Ergod. Th. \& Dynam. Sys., 17, 531-564 (1997). MR.1452179(99b:58137)

[3] Cawley, E., The Teichmüller space of an Anosov diffeomorphism of $T^{2}$, Inventiones Mathematicae, 112, 351-376 (1993). MR1213107 (94c:58141)

[4] Coullet, P. and Tresser, C., Itération d'endomorphismes et groupe de renormalisation, J. de Physique, C5, 25 (1978).

[5] Feigenbaum, M., The universal metric properties of nonlinear transformations, J. Stat. Phys., 21 (1979), 669-706. MR.555919 (82e:58072)

[6] Feigenbaum, M., Quantitative universality for a class of nonlinear transformations, J. Stat. Phys., 19 (1978), 25-52. MR0501179 (58:18601)

[7] Jacobson, M.V., Swiatek, G., Quasisymmetric conjugacies between unimodal maps. I. Induced expansion and invariant measures. Stony Brook preprint (1991).

[8] Jiang, Y., Asymptotic differentiable structure on Cantor set. Comm. in Math. Phys., 155 (3), 503-509 (1993). MR1231640(94e:58100)

[9] Jiang, Y., Renormalization and Geometry in One-Dimensional and Complex Dynamics. Advanced Series in Nonlinear Dynamics, Vol. 10, World Scientific Publishing Co. Pte. Ltd., River Edge, NJ (1996). MR1442953 (98e:58070)

[10] Jiang, Y., Smooth classification of geometrically finite one-dimensional maps. Trans. Amer. Math. Soc., 348 (6), 2391-2412 (1996). MR1321579 (96j:58054)

[11] Jiang, Y., On rigidity of one-dimensional maps. Contemporary Mathematics, AMS Series, 211, 319-431 (1997). MR1476995 (98h:58056)

[12] Jiang, Y., Metric invariants in dynamical systems. Journal of Dynamics and Differentiable Equations, Vol. 17 (1), 51-71 (2005). MR2157840(2006e:37070) 
[13] Jiang, Y., Teichmüller Structures and Dual Geometric Gibbs Type Measure Theory for Continuous Potentials, preprint (2008).

[14] Jiang, Y., Cui, G., Gardiner, F., Scaling functions for degree two circle endomorphisms. Contemporary Mathematics, AMS Series, 355, 147-163 (2004). MR2145061 (2006d:37067)

[15] Jiang, Y., Cui, G., Quas, A., Scaling functions, Gibbs measures, and Teichmüller space of circle endomorphisms, Discrete and Continuous Dynamical Systems, 5, no. 3, 535-552 (1999). MR.1696328 (2000f:37023)

[16] Lanford, O., Renormalization group methods for critical circle mappings with general rotation number, VIIIth International Congress on Mathematical Physics (Maresille, 1986), World Sci. Publishing, Singapore (1987), 532-536. MR.915597

[17] Mañé, R., Ergodic Theory and Differentiable Dynamics, Springer-Verlag, Berlin (1987). MR889254 (88c:58040)

[18] de Melo, W., van Strien, S., One-dimensional Dynamics. A series of Modern Surveys in Mathemaics, Springer-Verlag, New York (1993). MR1239171 (95a:58035)

[19] Pinto, A. A. and Rand, D. A., Renormalization gives all surface Anosov diffeomorphisms with a smooth invariant measure, submitted.

[20] Pinto, A. A. and Rand, D. A., Solenoid functions for hyperbolic sets on surfaces, Dynamics, Ergodic Theory and Geometry: Dedicated to Anatole Katok (ed. Boris Hasselblat) MSRI Publications, 54 (2007), 145-178. MR2369446(2010b:37083)

[21] Pinto, A. A. and Rand, D. A., Geometric measures for hyperbolic sets on surfaces. Stony Brook preprint, (2006).

[22] Pinto, A. A. and Rand, D. A., Rigidity of hyperbolic sets on surfaces, J. London Math. Soc., 71, 2 (2004), 481-502. MR2122440 (2005k:37051)

[23] Pinto, A. A. and Rand, D. A., Smoothness of holonomies for codimension 1 hyperbolic dynamics, Bull. London Math. Soc., 34 (2002), 341-352. MR1887706 (2003a:37037)

[24] Pinto, A. A. and Rand, D. A., Teichmüller spaces and HR structures for hyperbolic surface dynamics, Ergod. Th. Dynam. Sys. 22 (2002), 1905-1931. MR.1944410 (2004i:37055)

[25] Pinto, A. A. and Rand, D. A., Classifying $C^{1+}$ structures on dynamic fractals: 1 The moduli space for solenoid functions for Markov maps on train-tracks, Ergod. Th. Dynam. Sys. 15 (1995), 697-734. MR1346397 (97b:58094)

[26] Pinto, A. A., Rand, D. A., Ferreira, F., Fine structures of hyperbolic diffeomorphisms, Springer-Verlag Monograph (2008). MR2464147

[27] Pinto, A. A., Rand, D. A., Ferreira, F., Cantor exchange systems and renormalization, J. Differential Equations, 243 (2007), no. 2, 593-616. MR2371802 (2010d:37054)

[28] Pinto, A. A. and Sullivan, D., The circle and the solenoid. Dedicated to Anatole Katok on the Occasion of his 60th Birthday, DCDS-A 16, 2 (2006), 463-504. MR2226493 (2009b:37074)

[29] Rand, D. A., Global phase space universality, smooth conjugacies and renormalisation: 1. The $C^{1+\alpha}$ case, Nonlinearity, 1 (1988), 181-202. MR928952 (89g:58109)

[30] Schub, M., Global Stability of Dynamical Systems, Springer-Verlag (1987) MR869255 (87m:58086)

[31] Sullivan, D., Differentiable structures on fractal-like sets determined by intrinsic scaling functions on dual Cantor sets, Proceedings of Symposia in Pure Mathematics, 48, American Mathematical Society (1988). MR974329 (90k:58141)

[32] Veech, W., Gauss measures for transformations on the space of interval exchange maps, The Annals of Mathematics, 2nd Ser., 115, 2 (1982), 201-242. MR644019 (83g:28036b)

[33] Williams, R. F., Expanding attractors. Publ. I.H.E.S., 43 (1974), 169-203. MR0348794 $(50: 1289)$

Departamento de Matemática e LiaAd-InesC Porto LA, Faculdade de Ciências, Universidade do Porto, Rua do Campo Alegre, 687, 4169-007 Porto, Portugal

E-mail address: aapinto@fc.up.pt

Escola Superior de Techologia e de Gestão, Instituto Politécnico de Bragança, Campus de Santa Apolónia, Ap. 1134, 5301-854 Bragança, Portugal

E-mail address: jpa@ipb.pt

Instituto de Matemática, Facultad de Ingenieria, CC30, CP 11300, Universidad de la Republica, Montevideo, Uruguay

E-mail address: aldo@fing.edu.uy 